\title{
La educación a distancia: Una comparación con la educación presencial, en relación con la función del profesor, del estudiante y de los medios (Distance education: A comparison with traditional education in relation to the professor's role, the students, and its means)
}

\author{
Francisco J. Jardines \\ UANL, San Nicolás de los Garza, N.L., México, francisco.jardinesg@uanl.mx
}

Key words: Distance education, internet based education, online professor's role, information technology in education

\begin{abstract}
In this paper we present a description analysis of the differences between online vs. traditional education trough three issues: professor's role, student's role, and teaching means. In addition, we resume the historical evolution of the main differences and the implications of the learning process. Actually, the non-Internet based formation professor has to prepare in the new communication technologies; even thought resistance to change has been identified. However, efforts in this sense like training programs have been done through motivation conferences to these professors. The online students have distinguished by their academic success. We can mention some characteristics like solitaire capability to work and time administration, as well as perseverance and target focus. A deep comprehension of texts is an outstanding characteristic of an online student. The information technologies applied to the learning process have been evolved very fast in the last years, and studies have been demonstrated that the online education using these information technologies is not worse or better than the traditional class learning process. The results emphasize the interaction between the student and the objet of study, and with other students in the enforcement of the learning practices.
\end{abstract}

Palabras clave: Educación a distancia, educación por internet, función del profesor a distancia, tecnologías de la información en educación

Resumen. En este escrito se presenta una descripción, acompañada de algunas reflexiones sobre la comparación de la educación a distancia, con la educación presencial, en relación a la función del profesor, a la función del estudiante y a la función de los medios. De manera suscinta se establecen las diferencias más relevantes que se han presentado a la larga del tiempo y las implicaciones que afectan el proceso del aprendizaje. El docente sobre todo el

\section{Educación a Distancia}


que ya se formó y se preparó en tiempos previos al internet, ha tenido la necesidad de prepararse en el empleo de las nuevas tecnologías de la comunicación y aunque se ha presentado resistencia en este sentido, se han realizado esfuerzos en la capacitación y uso de los nuevos medios, apoyándose en el recurso de la motivación del docente. El estudiante de educación a distancia se distingue por varias características que se han observado en los que han logrado éxito en sus estudios. Estas características son entre otras, habilidades para trabajar de manera solitaria y administrar su tiempo, conducta enfocada hacia la meta y ser perseverante. Una característica sobresaliente es la comprensión profunda de los contenidos. Los medios de las tecnologías de información empleados en el proceso instruccional a distancia, han evolucionado rápidamente sobre todo en los últimos años, y se han obtenido resultados en el sentido que el aprendizaje construido por el estudiante a través de estos medios no es mejor ni peor que el aprendizaje de manera presencial. Se acentúa en este resultado la interacción del alumno con el objeto de estudio y con otros estudiantes en la ejecución de prácticas de aprendizaje.

\section{Introducción}

El propósito de este documento es describir algunas reflexiones sobre tres elementos que se han considerado fundamentales en el proceso de la instrucción en la educación a distancia, los cuales son: el profesor, el alumno y los medios instruccionales y como se advertirá su función ha evolucionado en mayor o menor grado con el trascurso del tiempo. La importancia del escrito radica en analizar la participación de estos tres elementos del proceso de aprendizaje de manera simultánea.

El papel del docente ha sido durante el devenir de la historia el eje central sobre el que ha descansado el desarrollo del proceso instruccional. Un factor de análisis es la adaptación del profesor a los cambios en la tecnología de la información y una de las explicaciones más calificadas para superar este cambio, en la capacitación y apoyo en el uso de las tecnologías, lo constituye la motivación en los profesores.

La función del alumno, también ha experimentado cambios en el devenir del tiempo y desde un papel pasivo, de mero receptor de contenido, ha ido transformándose en un constructor de sus ideas y criterios con base en ciertos factores como: habilidad para trabajar solo y la conducta orientada hacia la meta, y que junto a otros elementos se distingue y caracteriza al estudiante a distancia.

El papel de los medios instruccionales, en el transcurso de los años ha tenido modificaciones que se han acentuado de manera vertiginosa en los

\section{F. J. Jardines}


últimos veinte años. Desde la hoja impresa, la radio, la televisión y ahora el Internet y sus múltiples recursos de comunicación, ha propiciado la interacción del estudiante con el objeto de estudio, con el profesor y con otros estudiantes que se traduce en un aprendizaje de mayor comprensión y por más largo tiempo.

\section{Comparación de la educación a distancia, con la educación presencial, en relación a la función del profesor}

En la educación presencial la función del profesor es referida por Andersen (1979) como la función inmediata en educación superior y propuso la siguiente definición de profesor inmediato: "El profesor inmediato es conceptualizado como los comportamientos no verbales que reducen la distancia física y psicológica entre los maestros y los estudiantes" (p. 544). Andersen basaba su concepto en el contacto visual con los estudiantes, adoptando una postura corporal relajada, usando gestos y sonriendo mejoraba el afecto de los estudiantes hacia las prácticas fomentadas en el curso, el dominio de las cuestiones del curso y hacia el instructor del curso.

Gorham (1998), amplió la definición de los comportamientos de profesor inmediato, para incluir los comportamientos orales tales como hablar de las experiencias acerca de lo que ha ocurrido fuera del salón de clases, usando buen humor, llamando a los estudiantes por su nombre, elogiando el trabajo o los comentarios de los estudiantes. Gorham sugirió que las consecuencias de este tipo de conductas contribuyen significativamente al aprendizaje de los estudiantes afectados.

Sanders y Wiseman (1990) ampliaron esta relación para incluir aprendizaje cognitivo y conductual. Definieron el aprendizaje cognitivo como la cantidad que han aprendido los estudiantes en un curso. Definieron el aprendizaje conductual como la probabilidad de que los estudiantes pudieran realmente usar conductas, prácticas, o teorías estudiadas en el curso. Observaron importantes correlaciones positivas entre los comportamientos verbales y no verbales del profesor inmediato y el estudiante afectado, además de conductas y aprendizaje cognitivo.

\section{Educación a Distancia}


Después de revisar las anteriores definiciones de profesor inmediato o profesor tradicional, se tratará la idea del profesor a distancia y sus retos académicos. Aunque la educación a distancia ha incrementado su desarrollo, no todos los profesores han recibido bien el necesario cambio de paradigma para implementar con éxito el modelo en línea. Es indispensable revisar cómo diseñar y facilitar esta situación emergente que se presenta con los profesores.

Las demandas de los profesores que utilizan la tecnología en la enseñanza continúan incrementándose (Fink, 2002). En el aprendizaje en línea, los estudiantes y los maestros están separados por el tiempo y la distancia. El modelo de aprendizaje en línea, presenta un conjunto de retos. Los temas de políticas, desarrollo de procedimientos, administración, apoyo y acceso tecnológico, temas legales, buena disposición de los docentes, aprovechamiento y satisfacción del estudiante son amplificados en el aprendizaje en línea.

Herman (2004), afirmó que identificando e implementando los factores motivacionales, deberá ser fácil hacer aparecer cercana la visión de una clase virtual que será facilitada por un asistente, 0 incluso por el estudiante mismo, dejando innecesario al profesor como un objeto primario para fomentar el cambio.

Tetenbaum (1998) señaló que el incremento de la era de la información causó cambios en la educación y en los empleos. En el mercado actual, el empleado más valioso es la persona que aprende rápido, trabaja colaborativamente fuera de una supervisión cerrada y podría hacerlo bien en un ambiente que cambia rápidamente.

Para tratar de superar el empleo de las tecnologías de comunicación por los profesores, además de la oportunidad de capacitación y apoyo en el empleo de dichas tecnologías, el punto fundamental es trabajar en la motivación de los profesores. La motivación es una expresión de la conducta humana y es útil comprender su naturaleza. La conducta es una función determinada por la interacción de la persona y la situación, sucede por algo que ocurre dentro y fuera de la persona y ese algo es la motivación (Hersey, Blanchard \& Johnson, 2001). Estos autores también especifican que la conducta es la meta directa, y es típicamente motivada por el deseo de lograr un resultado particular.

\section{F. J. Jardines}


Para predecir lo que un individuo puede hacer, los líderes necesitan comprender qué motivos o necesidades producen ciertos tipos de conducta en un tiempo dado. Las teorías han analizado la motivación desde la perspectiva del comportamiento de la gente en ciertas líneas de conducta para satisfacer alguna necesidad específica. Maslow (1954) creó una jerarquía de cinco tipos básicos de necesidades motivacionales, incluyendo las necesidades fisiológicas, tales como comida, aire y agua, necesidades de seguridad (como albergue), necesidades sociales, necesidades de aprecio y de auto-realización. La jerarquía existe dentro de una escala continua, donde una vez que la persona ha satisfecho parcialmente al menos una necesidad baja entonces se siente motivado a trabajar en la siguiente necesidad. Clayton Alderfer (como se cita en Hughes, Ginnett \& Curphy, 1995) tomó un enfoque similar al de Maslow cuando desarrolló su teoría de Existencia, Relación y Crecimiento, ERG por sus siglas en inglés. Las necesidades de existencia comprenden a las necesidades fisiológicas y de seguridad de Maslow, las necesidades de relación son similares a las necesidades sociales, y las de crecimiento corresponden a las necesidades de aprecio y de auto-realización.

A diferencia de las teorías individuales de motivación, los enfoques organizacionales buscan factores ambientales dentro del lugar de trabajo para estudiar la motivación de los empleados. Durante la era industrial, una característica de la administración fue considerar a los trabajadores únicamente como objetos, que fácilmente podrían ser intercambiados, los trabajadores eran estrictamente supervisados y guiados hacia lo que tenían que hacer. Los empleados eran tratados simplemente como elementos de estabilidad económica (Reis \& Peña, 2001).

Argyris (como se cita en Hersey et al., 2001) determinó que la gente observó en el lugar de trabajo un desarrollo total como individuo. Frederich Herzberg (como se cita en Hersey et al.) trató de explicar en su teoría motivación = higiene (también conocida como Teoría de los Dos Factores o Teoría Bifactorial) la conducta humana desde la perspectiva de las necesidades, y muestra cómo las organizaciones podrían usar esta información para mejorar la estructura del lugar de trabajo para enfrentarse a esas necesidades. Herzberg expresó que la gente era motivada por factores de higiene (políticas, supervisión, condiciones de trabajo, dinero y otros) y por motivadores (realización, reconocimiento, responsabilidad en el trabajo, y

\section{Educación a Distancia}


otros). Herzberg determinó que los factores higiénicos fueron efectivos para evitar la insatisfacción en el trabajo, mientras que los motivadores impulsaron a la gente para un desempeño superior. Los factores higiénicos afectan la buena voluntad de las personas y los motivadores afectan la competencia de las personas (Hersey et al.). El investigador considera que la motivación es el factor que puede ayudar a mejorar el desempeño de los docentes en la realización del proceso enseñanza-aprendizaje del modelo de educación a distancia.

\section{Comparación de la educación a distancia, con la educación presencial, en relación a la función del estudiante}

Una de las situaciones que ha ocurrido en los últimos años es el incremento de estudiantes en cursos a distancia. Chute, Thompson y Hancock (1999) describieron a los estudiantes de post-secundaria del mañana, como estudiantes que hablarán múltiples lenguajes, vivirán por todo el mundo, se extenderán en campus remotos, en el gobierno, en lugares de negocios y directamente en sus casas. También una investigación de The University Continuing Education Association (1998) mostró una relación consistente entre el nivel de logros educativos y la tendencia a seguir oportunidades de educación continua.

De manera similar que en los negocios, la educación está siendo influenciada por la transformación de la era industrial a la era de la información (Patrinos, 2000). Los educadores tiene un doble reto: a) dar a los estudiantes las herramientas para estudiar a lo largo de la vida y b) desarrollar el proceso y procedimientos necesarios para obtener información y transformarla en un conocimiento útil personal y social (Chute et al., 1999).

De acuerdo con Phipps, Wellman y Merisotis (1998) para la eficacia en los logros del estudiante en la educación a distancia, se deben considerar las siguientes cuatro características del aprendizaje a distancia:

a) El proceso enseñanza-aprendizaje involucra actividades donde los estudiantes están a distancia del instructor;

b) Los medios involucran una combinación de estrategias que incluyen audioconferencias, audiocintas, computadoras, correo electrónico, fax, Internet, impresos, televisión y videocintas;

\section{F. J. Jardines}


c) Conocimiento y contenidos están disponibles a través de una variedad de fuentes; $y$

d) la entrega del curso deberá ser ofrecida en cualquier tiempo y en cualquier lugar.

La educación a distancia comenzó como un programa sin valor escolar en créditos. Servía como un suplemento de la modalidad tradicional y desde hace poco tiempo ha crecido rápidamente como un reemplazo de la modalidad universitaria escolar.

Una revisión de la literatura indicó que los estudiantes a distancia pueden diferir demográficamente de los estudiantes que asisten en un campus tradicional, además un estudiante a distancia se considera un estudiante no tradicional. La mayoría de los estudiantes a distancia de educación superior son profesionales que trabajan y que tienen edades de 25 a 50 años, con poco tiempo para asistir a cursos tradicionales (Moore \& Kearsley, 2005). La literatura también sugirió entre otras cosas que las mujeres eligen estudiar a distancia más que los hombres, de acuerdo con Burge (1998) y Harvey (1995).

La motivación, entre otros factores, desempeña un papel importante para seleccionar el método de aprender a distancia (Moore \& Kearsley, 2005). Otros investigadores como Powell, Conway y Ross (1990) utilizaron una metodología de auto-informe para identificar las características del éxito de estudiantes a distancia. Algunas de las características identificadas fueron a) la habilidad para trabajar solo, b) la conducta orientada hacia la meta, c) habilidades para administrar bien el tiempo y d) altos niveles de perseverancia.

El éxito en el estudio requiere la aptitud de auto-dirigirse y motivarse internamente. La carencia de estas habilidades puede afectar la satisfacción del estudiante y disminuir la probabilidad de completar un programa de aprendizaje a distancia (Moore \& Kearsley, 2005; Verduin \& Clark, 1991). Aunque estas características pueden no ser exclusivas de los estudiantes de aprendizaje a distancia, sí pueden ser identificadas como prerrequisitos cruciales para los estudiantes a distancia.

Morgan (1995) propuso otro método para describir al estudiante de aprendizaje a distancia. Él sugirió que los estudiantes a distancia pudieran ser clasificados por su enfoque para aprender el contenido del curso. Los dos

\section{Educación a Distancia}


enfoques son llamados: a) el enfoque superficial y b) el enfoque profundo. El enfoque superficial de aprendizaje lo identificó Morgan como una metodología no reflexiva. Los estudiantes que no están seguros de su aprendizaje tienden a enfocarse en memorizar hechos discretos con el objetivo de complementar tareas para satisfacer los requisitos del curso. Los estudiantes que hacen uso del enfoque profundo, por otra parte, se centran en una comprensión profunda del contenido del curso. Estos estudiantes tratan de enlazar la nueva información con los conceptos aprendidos previamente en un esfuerzo para adquirir una mejor comprensión del material del estudio. Los estudiantes que hicieron uso del enfoque profundo para estudiar, si estaban más concentrados y selectivos en su aprendizaje.

\section{Comparación de la educación a distancia, con la educación presencial, en relación a la función de los medios}

Gery (2002) ofreció un considerable número de ideas con base en el apoyo técnico en tiempo real de los usuarios de los sistemas en computadora. Sus observaciones fueron valiosas para la investigación actual, pués dirigió a sus diseños directamente y a otros tipos de apoyos educativos por computadora, considerando la necesidad de un análisis cuidadoso de conducta y desempeño. Lock (2002) enfatizó que los temas técnicos pueden ser importantes para el éxito de estudiantes e instructores en el aprendizaje en línea. Lock observó que los instructores deberán poner mayor atención para satisfacer a los estudiantes en línea y comunicarse más frecuentemente dado que las interacciones son más provechosas y ocurre más aprendizaje.

No todos los métodos instruccionales dan por sí mismos facilidad para el apoyo tecnológico, sin embargo muchos sí lo hacen. Kaupins (2002) estudió la preferencia de los estudiantes con respecto a varios métodos instruccionales, la lista de los métodos estudiados y su posición en la preferencia por los estudiantes se presenta en la Tabla 1. En este estudio aparece que los métodos preferidos por los estudiantes para aprender, son los más prácticos y participativos. Los métodos que involucran varios mecanismos de apoyo electrónico, son menos efectivos en el aprendizaje cuando no propician la participación activa del estudiante.

Kaupins se apoyó en la teoría andragógica como una razón para el bajo rango de los métodos con apoyos electrónicos, sugiriendo que los

\section{F. J. Jardines}


estudiantes no pudieron emplearlos en períodos de participación activa o en casos de estudios reales o experiencias de prácticas de aprendizaje.

Por su parte Bober y Dennen (2001) exploraron un aspecto de los conceptos del aprendizaje constructivista en ambientes en línea y determinaron el proceso por el cual el conocimiento es construido a través de la comunicación electrónica. Su trabajo es de gran valor en la investigación actual porque explica las bajas calificaciones del papel de los instructores en la interacción con los estudiantes y porque está centrado en el concepto de comprensión compartida o intersubjetividad, un elemento fundamental en las comunidades de aprendizaje establecidas.

Tabla 1

Métodos instruccionales y su nivel de preferencia

\begin{tabular}{lc}
\hline Método instruccional & Preferencia \\
\hline Prácticas de aprendizaje & 1 \\
Instrucción 1 a 1 & 2 \\
Casos de Estudio (reales) & 3 \\
Representación & 4 \\
Casos de Estudio (simulados) & 5 \\
Juegos & 6 \\
Lectura con preguntas & 7 \\
Prácticas en la Web & 8 \\
CD-ROM & 9 \\
Auto-estudio de materiales & 10 \\
Capacitación sensible & 11 \\
Televisión interactiva & 12 \\
Videos interactivos en la Web & 13 \\
Instrucción programada & 14 \\
Videos en la Web & 15 \\
Televisión (transmitida) & 16 \\
Tipos de lectura en la Web & 17 \\
Videos & 18 \\
Audio en la Web & 19 \\
Radio & 20 \\
\hline
\end{tabular}

Nota. Adaptado de "Trainer opinions of selected computer-based training methods", por G. Kaupins, 2002, Journal of Education for Business, 77, p. 321. Copyright 2002 Heldref Publications.

\section{Educación a Distancia}


En su análisis detallado de varias herramientas electrónicas utilizadas para apoyar la interacción de los estudiantes en la clase virtual Bober y Dennen hicieron énfasis en la necesidad para los instructores y diseñadores de programas y cursos en escoger esas herramientas cuidadosa y deliberadamente para obtener los objetivos de aprendizaje. Citaron por ejemplo, el uso del Chat Room (el tipo más común de comunicación sincrónica) para construir un sentido de comunidad.

Bober y Dennen (2001) también elaboraron un análisis bien estructurado de las características comparativas de las comunicaciones sincrónicas y asincrónicas. La más común de las comunicaciones sincrónicas es el chat en línea, lo más parecido a una discusión en clase presencial. El principal reto es ser genuino en una conversación en línea espontánea, sin embargo, debió ser algo tan sencillo como decidir qué gente podría tomar turnos para hablar, especialmente cuando los chats involucran gran número de participantes. En este caso, las ideas y conceptos ofrecidos por los estudiantes pueden ser difíciles de seguir, cuando algunos participantes dominan la conversación. Otros estudiantes pueden estar distraídos durante el chat. Otros pueden tener dificultades para empezar a engranarse con participantes quienes quieren pasar desapercibidos. En la comunicación asincrónica (por ejemplo correo electrónico, tablero de anuncios, lista de servicios o grupos de noticias), los participantes serán capaces de seguir más de una discusión y contribuir en las discusiones sin ser interrumpidos 0 esperar su turno. El obstáculo aquí, de acuerdo con Bober y Dennen está en que la comunidad se construye con más dificultad, porque los participantes están en control del orden en que ellos leen los mensajes recibidos, el tiempo requerido para responder y la potencial necesidad de numerosos mensajes para clarificar declaraciones y pensamientos de los demás participantes.

Prestera y Moller (2001) sugirieron que los estudiantes necesitan tener oportunidades para participar en trabajos de comunicación sincrónica y asincrónica. También recomendaron usar espacios de presentación virtual, conocidos como ambientes de aprendizaje intencional apoyados en computadora, bases de datos basados en la web donde los estudiantes puedan acceder a información virtual y de texto. Estos autores también recomendaron que a las discusiones apoyadas por el instructor para estudiantes a distancia se les deberá dar alta prioridad en la estructuración de los cursos, así los estudiantes tendrán tiempo para reflexionar en la

\section{F. J. Jardines}


información aprendida y para discutir con los otros participantes. Particularmente con los modos de comunicación asincrónica, los estudiantes tendrán más tiempo de pensar con respecto a los materiales instruccionales y responder de manera significativa tal como se hace en un ambiente de clase tradicional.

DeBard y Guidera (1999) presentaron un argumento fuerte para usar la comunicación asincrónica en clases electrónicas. Primero ellos afirmaron que la clase asincrónica puede ser independiente de tiempo y lugar y entonces constituye un contexto único de discusión. La segunda ventaja es que esta clase es apoyada por tecnología ya de uso común por estudiantes y maestros en la mayoría de las instituciones de educación superior. Su primer argumento de cualquier modo, fue que la comunicación asincrónica podría ampliar la eficacia del aprendizaje del estudiante porque corresponde a los 7 principios de la enseñanza efectiva desarrollados por Chickering y Reisser (como se cita en DeBard \& Guidera, 1999). Estos principios son:

1. Fortalecimiento de los contactos maestro alumno

2. Fortalecimiento de la cooperación entre estudiantes

3. Fortalecimiento del aprendizaje activo

4. Dar retroalimentación rápida

5. Enfatizar el tiempo en tareas

6. Comunicaciones con altas expectativas y

7. Respeto a los diversos talentos y maneras de conocimientos

Los ambientes de aprendizaje asincrónicos no están fuera de problemas, especialmente para estudiantes que viajan diariamente al trabajo y aquellos quienes trabajan tiempo completo fuera de la universidad, el acceso puede ser un problema en períodos 0 en lapsos de tiempo que inhiben la discusión. Algunos estudiantes no pueden hacer preguntas de lo que no comprenden respecto a los procesos o contenidos del curso porque están dudosos de recibir una respuesta rápida. La percepción de anonimato que impregna el Internet puede ser una ventaja para ciertos estudiantes con limitaciones físicas o culturales, 0 una desventaja generalmente si los estudiantes fomentan hacer comentarios negativos 0 de otro modo se comportan sin restricciones en la comunicación cara a cara. Finalmente, sin señales no verbales del lenguaje corporal, gestos, tono de voz y expresiones

\section{Educación a Distancia}


faciales disponibles con ellos en la comunicación cara a cara, los estudiantes pueden malinterpretar las declaraciones de los otros estudiantes.

\section{Conclusión}

Conforme a lo descrito en el desarrollo del documento, podemos señalar algunas ideas a manera de conclusiones:

-En los profesores preparados con anterioridad a la aparición del Internet, se ha presentado en algunos, resistencia para utilizar los medios actuales de las tecnologías de la comunicación, para lo que es necesario ofrecer capacitación y apoyo en el empleo de las tecnologías citadas de manera oportuna y trabajar en la motivación de los mismos profesores.

- En lo que concierne a los estudiantes que desarrollan cursos a distancia se ha observado que una gran parte son trabajadores, con edades de 25 a 50 años, con poco tiempo para asistir a cursos presenciales y con mayor participación de las mujeres que de los varones. Algunas de las características que los distinguen son: tienen habilidad para trabajar solos, lo hacen con base a resultados que se proponen lograr, administran de manera efectiva el tiempo y tienen alto grado de perseverancia.

- Los medios instruccionales, que se ofertan en base a las tecnologías de la comunicación, y que se han distinguido por sus rápidos cambios sobretodo en los últimos años, favorecen herramientas que proporciona el Internet y que ayudan a disponer de interacción con los objetos de estudio, con maestros no presenciales, con compañeros alumnos ubicados a distancia, que ayudan a la reflexión de los contenidos y solución de situaciones problemáticas en equipos a distancia, mejorando la comprensión y la aplicación de conocimientos en casos de estudios simulados y reales.

\section{Referencias}

Andersen, J. F., 1979. Teacher immediacy as a predictor of teaching effectiveness. En D. Nimmo (Ed.), Communication yearbook (pp. 543-559). New Brunswick, NJ, EE. UU.: Transaction Books.

Bober, M. J. \& Dennen, V. P., 2001. Intersubjectivity: Facilitating knowledge construction in online environments. Educational Media International, 38(4), 241-250.

\section{F. J. Jardines}


Burge, E., 1998. Gender in distance education. En C. C. Gibson (Ed.), Distance learners in higher education: Institutional responses for quality outcomes (pp. 25-45). Madison, WI, EE. UU.: Atwood Publishing.

Chute, A., Thompson, M. \& Hancock, B., 1999. The McGraw-Hill handbook of distance learning: A "how to get started guide" for trainers and human resources professionals. Nueva York, NY, EE. UU.: McGraw-Hill.

DeBard, R. \& Guidera, S., 1999/2000. Adapting asynchronous communication to meet the seven principles of effective teaching. Journal of Educational Technology Systems, 28(3) $219-230$

Fink, M. L., 2002. Faculty on the move: Rethinking faculty support services. Syllabus, 15(7), 27-29.

Gery, G. J., 2002. Task support, reference, instruction, or collaboration? Factors in determining electronic learning and support options. Technical Communication, 49(4), 420-427.

Gorham, J., 1988. The relationship between verbal teacher immediacy behaviors and student learning. Communication Education, 37(1), 40-53.

Harvey, C., 1995. Increasing course completion rates. Adults Learning, 6(6), 178-179.

Herman, T. L., 2004. The effect of an online and face-to-face course delivery model on achievement for students enrolled in two computer basics courses: Ethical and leadership considerations. (Disertación doctoral, Bowling Green State University, 2004). Dissertation Abstracts International, 66 (01), 107A. (UMI No. 3159606)

Hersey, P., Blanchard, K. H. \& Johnson, D. E., 2001. Management of organizational behavior: Leading human resources (8a. ed.). Upper Saddle River, NJ, EE. UU.: Prentice Hall.

Hughes, R. L., Ginnett, R. C. \& Curphy, G. J., 1995. Understanding and influencing follower motivation. En J. T. Wren (Ed.), The Leader's Companion: Insights on Leadership Through the Ages (pp. 327-338). Nueva York, NY, EE. UU.: Free Press.

Kaupins, G., 2002, julio/ agosto. Trainer opinions of selected computer-based training methods. Journal of Education for Business, 77(6), 319-323. Recuperado el 25 de mayo de 2006 de la base de datos de Wilson Web.

Lock, J. V., 2002. Laying the groundwork for the development of learning communities within online courses. Quarterly Review of Distance Education, 3(4), 395-408.

Maslow, A., 1954. Motivation and personality. New York, EE. UU.: Harper \& Row.

Moore, M. G. \& Kearsley, G., 2005. Distance education: A system view (2a. ed.). Belmont, CA, EE. UU.: Wadsworth Publishing.

Morgan, A. R., 1995. Improving student learning in distance education: Theory, research and practice. European Journal of Psychology of Education, 10(2), 121.

Patrinos, H. A., 2000, marzo. Market Forces in Education. European Journal of Education, $35(1), 61-80$

Phipps, R. A., Wellman, J. V. \& Merisotis, J. P., 1998. Assuring quality in distance learning: A preliminary review. Washington, DC, EE. UU.: Council for Higher Education Accreditation.

\section{Educación a Distancia}


Powell, R., Conway, C. \& Ross, L.,1990. Effects of student predisposing characteristics on student success. Journal of Distance Education, 5(1). Recuperado el 10 de julio de 2006 de: http://cade.athabascau.ca/vol5.1/8_powell_et_al.html

Prestera, G. E. \& Moller, L. A. , 2001. Facilitating asynchronous distance learning: Exploiting opportunities for knowledge building in asynchronous distance learning environments. Recuperado el 15 de octubre de 2005 de: http://www.mtsu.edul itconf/ proceed01/3.pdf

Reis, D. \& Peña, L., 2001. Reenginering the motivation to work. Management Decision, $39(8), 666-675$.

Sanders, J. A. \& Wiseman, R. L., 1990. The effects of verbal and nonverbal teacher immediacy on perceived cognitive, affective and behavioral learning in the multicultural classroom. Communication Education, 39(4), 341-352.

Tetenbaum, T. J., 1998, primavera. Shifting paradigms: From Newton to chaos. Organizational Dynamics, 26(4), 21-32.

The University Continuing Education Association, 1998. Lifelong learning trends: A profile of continuing higher education. Washington, DC, EE. UU.: University Continuing Education Association.

Verduin, J. R. \& Clark, T. A., 1991. Distance education: The foundations of effective practice. San Francisco, CA, EE. UU.: Jossey-Bass.

\section{F. J. Jardines}

